This is the accepted version of the following article:

Gramazio F., Lorenzoni M., Pérez-Murano F., Evangelio L., Fraxedas J.. Quantification of nanomechanical properties of surfaces by higher harmonic monitoring in amplitude modulated AFM imaging. Ultramicroscopy, (2018). 187. : 20 . 10.1016/j.ultramic.2018.01.013,

which has been published in final form at https://dx.doi.org/10.1016/j.ultramic.2018.01.013 (c) https://dx.doi.org/10.1016/j.ultramic. 2018.01.013. This manuscript version is made available under the CC-BY-NC-ND 4.0 license http://creativecommons.org/licenses/by-nc-nd/4.0/ 


\title{
Quantification of nanomechanical properties of surfaces by higher harmonic monitoring in amplitude modulated AFM imaging
}

\author{
Federico Gramazio $^{1}$, Matteo Lorenzoni ${ }^{2}$, Francesc Pérez-Murano ${ }^{2}$, Laura Evangelio ${ }^{2}$, \\ Jordi Fraxedas ${ }^{1}$ \\ ${ }^{1}$ Catalan Institute of Nanoscience and Nanotechnology (ICN2), CSIC and BIST, Campus UAB, \\ Bellaterra, 08193 Barcelona, Spain \\ ${ }^{2}$ Instituto de Microelectrónica de Barcelona (IMB-CNM, CSIC), Campus UAB, 08193 Bellaterra, \\ Barcelona, Spain \\ E-mail: jordi.fraxedas@icn2.cat, francesc.perez@imb-cnm.csic.es
}

\begin{abstract}
The determination of nanomechanical properties is an intensive topic of study in several fields of nanophysics, from surface and materials science to biology. At the same time, amplitude modulation force microscopy is one of the most established techniques for nanoscale characterization. In this work, we combine these two topics and propose a method able to extract quantitative nanomechanical information from higher harmonic amplitude imaging in atomic force microscopy. With this method it is possible to discriminate between different materials in the stiffness range of $1-3 \mathrm{GPa}$, in our case thin films of PS-PMMA based block copolymers. We were able to obtain a critical lateral resolution of less than $20 \mathrm{~nm}$ and discriminate between materials with less than a $1 \mathrm{GPa}$ difference in modulus. We show that within this stiffness range, reliable values of the Young Modulus can be obtained under usual imaging conditions and with standard dynamic AFM probes.
\end{abstract}

Keywords: atomic force microscopy, higher harmonics, nanomechanics, block copolymers

\section{Introduction}

The investigation of interactions between surfaces allows the understanding of a wide range of phenomena from friction to stability of colloidal particles in liquid media [1]. A theoretical understanding of surface forces was developed by Hertz [2], Derjaguin and Landau [3], Johnson, Kendall and Roberts[4] and Derjaguin, Muller and Toporov [5], to mention the most widely referred. From the experimental point of view, the invention of the atomic force microscope (AFM) in 1986 [6] allowed the study of near-surface mechanical properties at the nano-scale thanks to the possibility of measuring forces in the $\mathrm{pN}$ range, with a sensitivity and spatial resolution smaller than that provided by dynamical mechanical analysis (DMA) apparatus [7]. Several operation modes have been developed in order to improve the performance of AFM in the measurement of mechanical properties 
[8] including quasi-static (such as quasi-static indentation [9], force-volume mode [10], adhesion force mode [11]); dynamic in contact mode (force modulation [12], contact resonance [13], ultrasonic force microscopy [14]); and dynamic in intermittent contact mode (phase imaging [15], phase modulation [16], pulsed-force [17]).

Despite this variety of techniques, the extraction of elastic moduli and viscoelastic constants remains problematic due to the difficulty of correctly describing the tip-surface contact area and the lack of accurate models which can describe the contact mechanics appropriately. Within the panorama of intermittent contact techniques, one of the most widely used is the amplitude modulation (AM-AFM) in repulsive mode, where the tip is periodically in contact with the sample surface. When the tip interacts with the sample surface, harmonic signal components are induced to the cantilever motion due to the non-linear character of the interaction [18]. These components and the force acting on the cantilever can be expressed in terms of Fourier series, as exhaustively described in theoretical works [18-20]. Although the higher harmonic amplitudes are well below $1 \mathrm{~nm}$, such amplitudes can be monitored with a reasonable signal-to-noise ratio when they are amplified by a flexural eigenmode. In our case, we chose to detect the $6^{\text {th }}$ harmonic oscillation amplitude because it is in close proximity with the $2^{\text {nd }}$ flexural eigenmodes. Considerable theoretical effort has been made in the last few years to describe and understand the potential use of higher harmonics [21-23] and its functional dependence on relevant parameters such as cantilever force constant $\left(k_{\mathrm{c}}\right)$, tip radius $(R)$, free oscillation amplitudes $\left(A_{0}\right)$ and sample stiffness $(E)$ [24,25]. It is well known that only a few amplitude signals are detectable when the tip-sample interaction is kept gentle i.e. $100 \mathrm{pN}$ [26]. To overcome this limitation, we extrapolated the most suitable experimental conditions in order to enhance the higher harmonic signal by carrying out simulations with the Virtual Environment for Dynamic AFM (VEDA) software [27]. The focus was on the experimental quantification of sample surface Young modulus $(E)$ analyzing the amplitude of the $6^{\text {th }}$ higher harmonic and comparing it with the simulations. This is the first time that experiments have been conducted to estimate surface mechanical properties in the optimum conditions for this method. The samples chosen for the $E$ quantification were block copolymers (BCPs) thin films [28,29].

\section{Materials and Methods}

\subsection{Simulations}

Simulations were performed using the VEDA open code, which takes into account the dynamics of oscillating rectangular cantilevers with multiple eigenmodes [27]. The frequencies, stiffness and quality factors of the 2nd flexural eigenmode have been approximated by the known relationships $6.27 f_{0}, 6.27^{2} k_{\mathrm{c}}$ and $6.27 Q$, respectively, corresponding to a massless tip, where $f_{0}$ and $Q$ stand for the 
resonance frequency (fundamental mode or first eigenmode) and the quality factor, respectively [30]. We have used the Derjaguin-Muller-Toporov (DMT) model to describe the tip-surface interaction [5] and the tip was approximated by a hemisphere with a well-defined radius $R=5 \mathrm{~nm}$. For simplicity, neither viscoelastic nor capillary forces were considered [25].

\subsection{Experimental}

AFM imaging of topography, fundamental mode phase and $6^{\text {th }}$ higher harmonic were performed in the repulsive mode in order to enhance the $6^{\text {th }}$ higher harmonic amplitude $\left(A_{6}\right)$ signal well above the noise level of the employed electronic. A commercial AFM was employed, hosted in a homemade controlled humidity environment. Commercial TESP SS (Bruker) rectangular microfabricated silicon cantilevers with ultrasharp silicon tip $(2<R<5 \mathrm{~nm})$ were used with the nominal values $k_{\mathrm{c}} \approx 42 \mathrm{~N} / \mathrm{m}$ and $f_{0} \approx 300 \mathrm{kHz}$. The amplitude of the higher harmonics was registered using a digital signal processing (DSP) dual phase, wide bandwidth external lock-in amplifier. The use of an external lockin allowed us to improve the signal-to-noise ratio and the lowest limit of detectable values within the range of $70-600 \mu \mathrm{V}$ as evidenced by a resulting higher resolution at lower free amplitude values as compared to those achievable with an internal lock-in. The laser spot was positioned at its revealed maximum intensity, coinciding with the position of the tip along the cantilever, and the dynamical $k_{\mathrm{c}}$ was calibrated performing thermal tune measurement applying Sader's corrections [31-32]. In order to convert the $6^{\text {th }}$ higher harmonic amplitude values from $\mu \mathrm{V}$ to $\mathrm{nm}$, the laser-detector deflection sensitivity was calibrated by performing a ramp in tapping mode on a sapphire calibration sample. AFM measurements were always performed at relative humidity below $10 \%$, in order to reduce the capillarity condensation at the tip-surface interaction as much as possible.

\subsection{Self-assembled Block Copolymers samples}

BCPs are macromolecules built from more than one species of monomers with inter-monomer covalent bonding which tend to segregate due to the repulsion between different blocks and undergo a separation phase with controllable dimensions and functionalities [33-34]. These materials show periodic structures with a characteristic width or pitch and have been found interesting for high volume manufacturing in the semiconductor industry $[35,36]$. The fact that low cost and nanometer scale features at high throughput are obtainable by directed self-assembly (DSA) is attracting interest in the whole fields of lithography and surface patterning. The samples employed in the present work were made using poly(styrene-b-methylmethacrylate) (PS-b-PMMA 50:50, $\mathrm{M}_{\mathrm{n}}=79 \mathrm{~kg} \cdot \mathrm{mol}^{-1}$, PDI $=1.13$ ) provided by Arkema and assembled on a polystyrene based (PS-OH) brush layer. BCP was dissolved in a $1.5 \%(\mathrm{w} / \mathrm{w})$ solution of propylene glycol methyl ether acetate (PGMEA). Thin films made of this BCP form lamellae with a nominal pitch of $37 \mathrm{~nm}$. In order to graft the brush layer, the 
prepared surfaces were annealed for $5 \mathrm{~min}$ at $230{ }^{\circ} \mathrm{C}$ in nitrogen atmosphere. The remaining ungrafted brush was removed with PGMEA rinsing. Above this brush layer, the L37 solution was spin-coated and annealed a second time for $10 \mathrm{~min}$ at $230{ }^{\circ} \mathrm{C}$ in nitrogen atmosphere, to induce the lamellae selfassembly with a nominal pitch of $37 \mathrm{~nm}$.

In order to selectively modify the stiffness of one of the phases of the block co-polymer samples, a Sequential Infiltration Synthesis (SIS) process was applied [37]. Alumina, obtained from binary reactions of trimethylaluminium (TMA) and water at $135{ }^{\circ} \mathrm{C}$, was obtained at the end of the infiltration process which took place in two steps: first, TMA was placed in a vacuum chamber for 60 $\mathrm{s}$ and then, once purged, water was introduced. This entire sequence was repeated for 5 times, and each time the infiltration process not only covered the top domain but the whole polymer domains, resulting in a global improvement of resistant hardness, leading to high-aspect-ratio features in a substrate [38-41].

\section{Results and Discussion}

In a previous work we have explored the functional dependence of $A_{6}$ as a function of $R, A_{0}, E$ and $k_{\mathrm{c}}$ and shown that $A_{6}$ follows a universal gun-shape curve, with coefficients that depend on the values of the mentioned parameters [24,25]. The functional dependence can help to determine the best experimental conditions to quantify, e.g., E. The simulations shown in Fig. 1 indicate the evolution of $A_{6}$ as a function of the mentioned parameters and are useful to select the experimental conditions aimed to maximize the signal and the sensitivity in order to extract experimental values of the Young's modulus. Figure 1a shows the dependence of $A_{6}$ as a function of the $z$ distance (the distance between the cantilever base and the sample surface). $A_{6}(z)$ increases upon entering the repulsive region (dashed vertical green line) and reaches its maximum (blue vertical line) around $A_{0} / 4$. Most of the experiments presented in this work have been performed with an amplitude set point $\left(A_{\mathrm{sp}}\right)$ of $50 \%$ of $A_{0}$ (dashed red line), and under this condition $A_{6}$ is close to its maximum value.

The evolution of $A_{6}$ as a function of selected values of $k_{\mathrm{c}}$ is shown in Fig. 1b. Note that the amplitude decreases for increasing cantilever spring constant values, approximately following a $1 / k_{\mathrm{c}}$ dependence $[22,25]$. From the figure we observe that the value of $A_{6}$ is not very much dependent on the values of $k_{\mathrm{c}}$ for $k_{\mathrm{c}}>20 \mathrm{~N} / \mathrm{m}$. Figures $1 \mathrm{c}$ and $1 \mathrm{~d}$ show the dependence of $A_{6}$ as a function of $E$ for $k_{\mathrm{c}}=0.1 \mathrm{~N} / \mathrm{m}$ (Fig. 1c) and $k_{\mathrm{c}}=26 \mathrm{~N} / \mathrm{m}$ and $k_{\mathrm{c}}=42 \mathrm{~N} / \mathrm{m}$, respectively (Fig. 1d), to cover a wide range of $k_{\mathrm{c}}$ values. From the curves it becomes evident that the sensitivity (expressed in terms of the slope of the curve and represented in Figs. 1e and 1f, respectively) is highest for $\mathrm{E}<5 \mathrm{GPa}$, which is the region of interest for the polymers selected in this work. Above $E \approx 10 \mathrm{GPa}$ the slope becomes increasingly smaller which indicates that standard dynamic mode cantilevers would be not indicated for the quantitative 
determination of the $E$ values. From Fig. $1 \mathrm{~b}$ one would be tempted to choose the softest cantilevers, since the predicted $A_{6}$ signal is larger. However, very soft cantilevers are unstable in the repulsive mode due to torsional deformations and exhibit lower $Q$ values. In our case we have selected the 42 $\mathrm{N} / \mathrm{m}$ cantilevers because of their mechanical stability, larger $Q$ values and since the predicted $A_{6}$ signals are not significantly smaller than those corresponding to the intermediate cantilevers (ca. 20 $\mathrm{N} / \mathrm{m}$ ). Although a clear disadvantage when using stiffer cantilevers is the associated lower accuracy in the estimation of the actual $k_{\mathrm{c}}$ value using thermal tune calibration methods and the resulting lower oscillation amplitudes, the fact that the dependence of $k_{\mathrm{c}}$ on the value of $E$ is low mitigates the uncertainty on its quantification.

However, and as will be shown next, the lower values obtained from using a stiffer cantilever can be compensated by increasing the free oscillation amplitude $A_{0}$. This is shown in Figs. $1 \mathrm{~g}\left(k_{\mathrm{c}}=0.1 \mathrm{~N} / \mathrm{m}\right)$ and $1 \mathrm{~h}\left(k_{\mathrm{c}}=26 \mathrm{~N} / \mathrm{m}\right.$ and $\left.k_{\mathrm{c}}=42 \mathrm{~N} / \mathrm{m}\right)$, where it is observed that $A_{6}$ increases linearly with $A_{0}$ in the 40 $80 \mathrm{~nm}$ range. $A_{0}$ appears to be a crucial working parameter directly linked to the peak force exerted on the sample: higher harmonics increase monotonically with $A_{0}$ and, in this range of values, are excited above the noise level without plastic deformation, despite the observation of large deformations as a free amplitude of $80 \mathrm{~nm}$ is approached. As the free amplitude was increased the dynamic excitation of the cantilever changed. To have an idea of how tip-sample interaction changes, an experiment under repulsive regime was considered, at a constant set-point value (50\%). Higher peak interaction force was exerted as $A_{0}$ increased. The peak interaction force, $\mathrm{F}_{\text {peak }}$, can be expressed as [42]:

$$
F_{\text {peak }}=1.4 /\left[\left(E^{*} \sqrt{R}\right)^{-1 / 4}\left(Q / k_{c}\right)^{3 / 4} A_{0}^{-9 / 8}\right]
$$

where $E^{*}$ is the effective tip-sample elastic modulus. Equation 1 predicts $\mathrm{F}_{\text {peak }}$ ranging from $3.4 \mathrm{nN}$ $\left(A_{0}=40 \mathrm{~nm}\right)$ to $6.3 \mathrm{nN}\left(A_{0}=70 \mathrm{~nm}\right)$ using $R=5 \mathrm{~nm}, k_{c}=42 \mathrm{~N} / \mathrm{m}, Q=600$ and $E^{* \approx} 2 \mathrm{GPa}$, since the Young's modulus corresponding to silicon is much larger than the value corresponding to the PS and PMMA polymers. Within this range of forces, the DMT model predicts a deformation in the range of $3-8 \mathrm{~nm}$, as found experimentally (see Fig. 2a). This range of free amplitude values is higher than those usually employed in force reconstruction experiments $[43,44]$. In our case, working at higher amplitudes allowed us to obtain a clearer detectable signal and compare the resulting trends with the simulations.

\subsection{PS - LDPE}

As a first step, the $6^{\text {th }}$ higher harmonic signal was studied on a Bruker's calibration sample of PS and low density polyethylene (LDPE), having nominal $E$ values of 2.0 and $0.1 \mathrm{GPa}$, respectively. The morphology of the surface consisted of a PS surface with LDPE semispherical micro-droplets. This 
kind of sample is a good starting point because the two phases are easily distinguishable in the topography (Fig. 2a), the phase channel (Fig. 2b) and also from the $6^{\text {th }}$ higher harmonic amplitude image (Fig. 2c) because of the large difference in Young's moduli (about $2 \mathrm{GPa}$ ) between the two phases. We were also able to verify that increasing $\mathrm{A}_{0}$ exerts higher forces inducing an increased deformation, as can be seen in the topography (Fig. 2a). It can be clearly observed that the droplet of LDPE at $A_{0}=70 \mathrm{~nm}$ (right half) shows a higher deformation than the one at $A_{0}=40 \mathrm{~nm}$ (left half). Despite the deformation, the theoretical values agreed with the experimental ones, as shown in Fig. 2e, confirming that the DMT model $[5,45]$ was also still valid at these amplitudes ranges.

The images were analyzed with the Gwyddion software [46] applying a threshold masking of the $6^{\text {th }}$ higher harmonic image according to the Otsu method [47], in order to discriminate the signal value from the two materials (Fig. 2d). The trend of $A_{6}$ mean values, taken from the different phases at different $A_{0}$, allows a clearer comparison with the simulations (Fig. 1h). The experimental results for PS lay between the two simulations performed for $E=2 \mathrm{GPa}$ and $E=1.8 \mathrm{GPa}$, while those for the LDPE lay between the simulations for $E=0.1 \mathrm{GPa}$ and $E=0.2 \mathrm{GPa}$. The obtained results and their indetermination were perfectly compatible with other works that have performed analogous measurements on the same kind of sample using other methods such as Force reconstruction [43] and nanomechanical spectroscopy [44].

\subsection{PS-b-PMMA}

The second type of sample investigated was a PS-b-PMMA self-assembled film with vertical lamellae and with a $37 \mathrm{~nm}$ pitch. The topography and phase images of this sample, measured at $A_{0}=70 \mathrm{~nm}$, are shown in Figs. $3 \mathrm{a}$ and $3 \mathrm{~b}$, respectively, showing a characteristic fingerprint distribution. In the topography image the two phases are distinguishable: PS and PMMA correspond to the darker and brighter parts, respectively, due to the different stiffness. The good contrast observed in Figs. 3a and $3 \mathrm{~b}$ is also visible in the $\mathrm{A}_{6}$ channel (Fig. 3c), which reliably replicates the topography and phase images. The $E$ values of the two phases of this sample had been measured previously [48] by PeakForce quantitative nanomechanical mapping (QNM), enabling a comparison of the two methods. The $E$ values obtained here for PS and PMMA are $1.97 \pm 0.21$ and $3.10 \pm 0.73 \mathrm{GPa}$, respectively. The simulation shown in Fig. 3d shows a good agreement between the experimental values matching the results previously obtained with QNM technique [48]. The experimental results lay between the simulations performed for $E=1.6 \mathrm{GPa}$ and $2 \mathrm{GPa}$ for PS and $2.4 \mathrm{GPa}$ and $3 \mathrm{GPa}$ for the PMMA, respectively, within the indetermination on the QNM results. However, the $A_{6}$ values at $A_{0}=80 \mathrm{~nm}$ become less reliable; at this free amplitude the surface of the sample started to deform plastically. Notwithstanding the lower difference of Young modulus $(\Delta E \approx 1 \mathrm{GPa})$ and the small dimensions of 
the block copolymers phases $(18 \mathrm{~nm})$, we obtained a good contrast that allows both the discrimination of both phases and a quantitative estimation of the corresponding $E$ values.

Another parameter that has an important effect on evolution of $A_{6}$ is the tip radius, as has been previously discussed [24,25]. In this case $A_{6}$ increases monotonically for increasing $R$ values, hence it becomes mandatory the monitoring of the evolution of $R$. In our case we have compared the topographies of the first image of the experiments $\left(A_{0}=40 \mathrm{~nm}\right)$ with the last one, after 5 images $\left(A_{0}=70\right.$ nm). Figure 4 shows the comparison of the corresponding cross sections where no significant change is observed. We can thus conclude that in this case the tip radius was constant so that the increase of $A_{6}$ can be safely attributed to the increase in $A_{0}$ and not to changes in $R$.

\subsection{PS-b-PMMA with modified PMMA by SIS}

The third kind of sample was a PS-b-PMMA self-assembled film with vertical lamellae and pitch of $37 \mathrm{~nm}$, exposed to SIS treatment in order to induce an increase of the stiffness of the PMMA phase. The use of SIS process is very interesting for pattern transfer: the infiltered phase becomes more resistant to etching, and in particular, the etching selectivity with silicon increases, allowing to increase the final aspect ratio of the transferred features. However, it is also important to ensure that the infiltered blocks have good mechanical properties, to avoid collapsing of the infiltered features when the non infiltered block is removed.

Previous measurements of the $E$ value of infiltered PS-b-PMMA was measured by QNM [48], obtaining a value of $3.6 \pm 0.4 \mathrm{GPa}$ for the PMMA phase. Using higher harmonic detection, we obtain the same information but with standard dynamic mode AFM imaging conditions and at lower surface deformation. The morphology of the fingerprint patterns does not change after the SIS cycles (Figs. 4a and $4 \mathrm{~b}$ ). Images of the $A_{6}$ channel (Figs. $4 \mathrm{c}$ and $4 \mathrm{~d}$ ) show that the resolution and the contrast are good and allow the two phases to be distinguished. The analysis using VEDA simulations (Fig. 4e) indicates that the $E$ values for the PMMA lay between 3 and $3.6 \mathrm{GPa}$, while those for the PS increases compared with the pristine PS-b-PMMA sample and lay above the simulation with $E=2 \mathrm{GPa}$, in accordance with the results obtained with QNM. It is then confirmed that the $A_{6}$ detection method presents sufficient sensitivity to detect changes of Young's modulus of around $0.5 \mathrm{GPa}$.

\section{Conclusions}

We have developed a method to determine the Young's modulus of nanostructured materials based on standard AFM tapping mode cantilever and easily available electronics. Under the same imaging 
conditions usually employed in dynamic AFM mode, we were able to reliably measure the amplitude of $6^{\text {th }}$ higher harmonic of the first resonance mode at free oscillation amplitudes of $40-80 \mathrm{~nm}$. VEDA simulations are used to analyze the dependence of imaging parameters on the values of the higher harmonics and surface properties, paving the way to select the proper conditions to optimize the measurements. We have obtained a good match between simulations and experimental values for polymeric samples in the low GPa range (2-4 GPa) scanned at high resolution, resolving features down to $17 \mathrm{~nm}$ width. Specifically, lamellar block copolymers with nominal pitch of $37 \mathrm{~nm}$ were characterized using the $6^{\text {th }}$ higher harmonic detection method. The results are in accordance with those reported by other methods such as PeakForce QNM, force reconstruction or nanomechanical spectroscopy, and with a comparable uncertainty. Moreover, simulations indicate that there is no need to use different tips for different ranges of Young modulus as required with PeakForce and Force volume techniques. Using a standard set-up has the advantage of analyzing the direct signal of the photodiode (amplitude of $6^{\text {th }}$ higher harmonic) and all the characteristics mentioned above, thus making it a possible alternative or a valid complement to other nanomechanical measuring approaches.

\section{Acknowledgements}

This work was performed within the aim4np project, which was supported by the EC through a grant (contract Nr. 309558) within the 7th Frame-work Program and within the framework of the PhD program in Physics of the Autonomous University of Barcelona. The ICN2 is funded by the CERCA programme / Generalitat de Catalunya. The ICN2 is supported by the Severo Ochoa programme of the Spanish Ministry of Economy, Industry and Competitiveness (MINECO, grant no. SEV-2013-0295). Laura Evangelio acknowledges the Ministry of Education of Spain for her FPU grant (FPU 13/03746). All polymeric materials have been supplied by Arkema. Additional financial support from Nanointegra project (TEC2015-69864-R) is also acknowledged.

\section{References}

[1] J. N. Israelachvili, Intermolecular and Surface Forces, 3rd ed., Elsevier, Amsterdam, Netherlands, 2011.

[2] H. J. Hertz, Reine Angew. Math. 92 (1881) 156-171.

[3] B. Derjaguin, L. Landau, Prog. Surf. Sci. 43 (1993) 30-59. 
[4] K. L. Johnson, K. Kendall, A. D. Roberts, Proc. R. Soc. A Math. Phys. Eng. Sci. 324 (1971) 301313.

[5] B. V. Derjaguin, V. M. Muller, Y. P. Toporov, J. Colloid Interface Sci. 53 (1975) 314-326.

[6] G. Binnig, C. F. Quate, Phys. Rev. Lett. 56 (1986) 930-933.

[7] K. P. Menard, Dynamic Mechanical Analysis: A Practical Introduction, $2^{\text {nd }}$ ed., CRC Press, 2008.

[8] D. Passeri, M. Rossi, E. Tamburri, M. L. Terranova, Anal. Bioanal. Chem. 405 (2013) $1463-$ 1478.

[9] J. Fraxedas, S. Garcia-Manyes, P. Gorostiza, F. Sanz, Proc. Natl. Acad. Sci. U. S. A. 99 (2002) $5228-5232$.

[10] K.-S. Kim, H, Wang, Q. Zou, Am. Control Conf. (2009) 991-996.

[11] K. O. Van der Werf, C. A. J. Putman, B. G. De Grooth and J. Greve, Appl. Phys. Lett. 65 (1994) 1195-1197.

[12] P. Maivald, H. J. Butt, S. A. C. Gould, C. B. Prater, B. Drake, J. A. Gurley, V. B. Elings and P. K. Hansma, Nanotechnology 2 (1991) 103-116.

[13] U. Rabe, S. Amelio, M. Kopycinska, S. Hirsekorn, M. Kempf, M. Göken and W. Arnold, Surf. Interface Anal. 33 (2002) 65-70.

[14] K. Yamanaka, A. Noguchi, T. Tsuji, T. Koike and T. Goto, Surf. Interface Anal. 27 (1999) 600606.

[15] J. Tamayo and R. Garcia, Langmuir 7463 (1996) 4430-4435.

[16] T. Fukuma, J. I. Kilpatrick and S. P. Jarvis, Rev. Sci. Instrum. 77 (2006) 123703.

[17] C. A. Rezende, L.-T. Lee and F. Galembeck, Langmuir 25 (2009) 9938-9946.

[18] R. V. Stark, W. M. Heckl, Surf. Sci. 457 (2000) 219-228.

[19] U. Dürig, New J. Phys. 2 (2000) 5.

[20] O. Sahin, C. F. Quate, O. Solgaard, A. Atalar, Phys. Rev. B 69 (2004) 165416.

[21] R. W. Stark, W. M. Heckl, Rev. Sci. Instrum. 74 (2003) 5111-5114.

[22] F. Giessibl, Surf. Interface Anal. 38 (2006) 1696-1701. 
[23] S. Santos, V. Barcons, J. Font, A.Verdaguer, Beilstein J. Nanotechnol. 5 (2014) 268-277.

[24] J. Fraxedas, F. Pérez-Murano, F. Gramazio, M. Lorenzoni, E. Rull Trinidad, U. Staufer, Proc. SPIE 9636 (2015) 963600.

[25] F. Gramazio, M. Lorenzoni, F. Pérez-Murano, E. Rull Trinidad, U. Staufer, J. Fraxedas, Beilstein J. Nanotechnol. 8 (2017) 883-891.

[26] K. Gadelrab, S. Santos, J. Font and M. Chiesa, Nanoscale 5 (2013) 10776.

[27] D. Kiracofe, J. Melcher, A. Rahman, Rev. Sci. Instrum. 83 (2012) 013702.

[28] S. Y. Chou, P. R. Krauss, P. J. Renstrom, Science 272 (1996) 85-87.

[29] P. Mansky, P. Chaikin, E. L. Thomas, J. Mater. Sci. 30 (1995) 1987-1992.

[30] R. Garcia, E. T. Herruzo, Nat. Nanotechnol. 7 (2012) 217-226.

[31] T. E. Schäffer, Nanotechnology 16 (2005) 664-670.

[32] J. E. Sader, J. Lu and P. Mulvaney, Effect of cantilever geometry on the optical lever sensitivities and thermal noise method of the atomic force microscope Rev. Sci. Instrum. 85 (2014) 113702.

[33] M. Seul, D. Andelman, Science 267 (1995) 476-483.

[34] F. S. Bates, G. H. Friedickson, Annu. Rev. Phys. Chem. 41 (1990) 525-557.

[35] B. G. Liu, C. S. Thomas, G. S. W. Craig and P. F. Nealey, Adv. Funct. Mater. 20 (2010) 12511257.

[36] B. Rathsack, M. Somervell, M. Muramatsu, K. Tanouchi, T. Kitano, E. Nishimura, K. Yatsuda, S. Nagahara, H. Iwaki, K. Akai, M. Ozawa, A. R. Negreira, S. Tahara, K. Nafus. Proceedings SPIE 8682 (2013) 86820K. DOI: 10.1117/12.2012018.

[37] M. Lorenzoni, L. Evangelio, M. Fernández-Regúlez, C. Nicolet, C. Navarro, and F. PérezMurano, J. Phys. Chem. C 121 (2017) 3078-3086. DOI: 10.1021/acs.jpcc.6b11233

[38] B. Q. Peng, Y. Tseng, S. B. Darling and J. W. Elam, Adv. Mater. 22 (2010) 5129-5133.

[39] M. Biswas, J. A. Libera and S. B. Darling, Chem. Mater. 26 (2014) 6135-6141.

[40] M. Biswas, J. A. Libera and S. B. Darling J. Phys. Chem. C 119 (2015) 14585-14592.

[41] R. Ruiz, L. Wan, J. Lille, K. C. Patel, E. Dobisz, D. E. Johnston, K. Kisslinger, C. T. Blackless, J. Vac. Sci. Technol. B, 30 (2012) 06F202. 
[42] S. Hu and A. Raman, Appl. Phys. Lett. 91 (2007) 123106.

[43] A. F. Payam, D. Martín-Jiménez, R. Garcia, Nanotechnology 26 (2015) 185706.

[44] E. T. Herruzo, A. P. Perrino, R. Garcia, Nat. Commun. 5 (2014) 3126.

[45] B. Cappella, Mechanical Properties of Polymers Measured through AFM Force-Distance Curves, Springer, Switzerland, 2016.

[46] D. Necas, P. Klapetek, Cent. Eur. J. Phys. 10 (2012) 181-188.

[47] N. Otsu, IEEE Trans. Syst. Man. Cybern. SMC-9 (1979) 62-66.

[48] M. Lorenzoni, L. Evangelio, S. Verhaeghe, C. Nicolet, C. Navarro, F. Pérez-Murano, Langmuir 31 (2015) 11630-11638. 

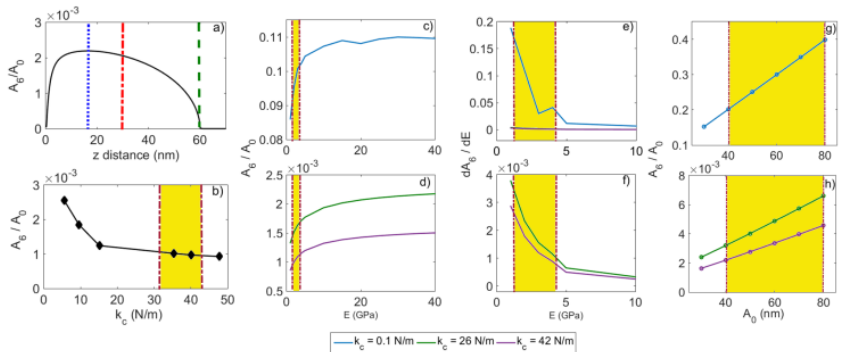

Figure 1. (a) Simulated dependence of $A_{6}$ normalized to $A_{0}$ as a function of the distance (z) between the cantilever base and the surface of the sample at $A_{0}=60 \mathrm{~nm}$ : the discontinuous green vertical line shows the threshold of the repulsive regime, the discontinuous red line represents the $z$ distance at $A_{\mathrm{sp}}$ $=0.5 A_{0}$ and the blue line represents the $z$ distance at which $A_{6}$ reaches its maximum. (b) Simulation of the dependence of $A_{6}$ normalized to $A_{0}$ as a function of $k_{\mathrm{c}}$, using selected nominal values (see ref. [25] for details). Evolution of $A_{6}$ normalized to $A_{0}$ as a function of $E$ for (c) $k_{\mathrm{c}}=0.1 \mathrm{~N} / \mathrm{m}$ (continuous blue line) and (d) $k_{\mathrm{c}}=26 \mathrm{~N} / \mathrm{m}$ (continuous green line) and $\mathrm{kc}=42 \mathrm{~N} / \mathrm{m}$ (continuous magenta line). The derivative of curves in (c) and (d) are shown in (e) and (f), respectively, in order to highlight the slopes. Evolution of $A_{6}$ normalized to $A_{0}$ as a function of $A_{0}$ for (c) $k_{\mathrm{c}}=0.1 \mathrm{~N} / \mathrm{m}$ (blue dots) and (d) $k_{\mathrm{c}}$ $=26 \mathrm{~N} / \mathrm{m}$ (green dots) and $k_{\mathrm{c}}=42 \mathrm{~N} / \mathrm{m}$ (magenta dots). The yellow highlighted areas represent the working range: $E=0.1 \mathrm{MPa}-4.7 \mathrm{GPa}, k_{\mathrm{c}}=30-43 \mathrm{~N} / \mathrm{m}$. All $A_{6}$ values have been calculated at $50 \%$ of $A_{0}$.
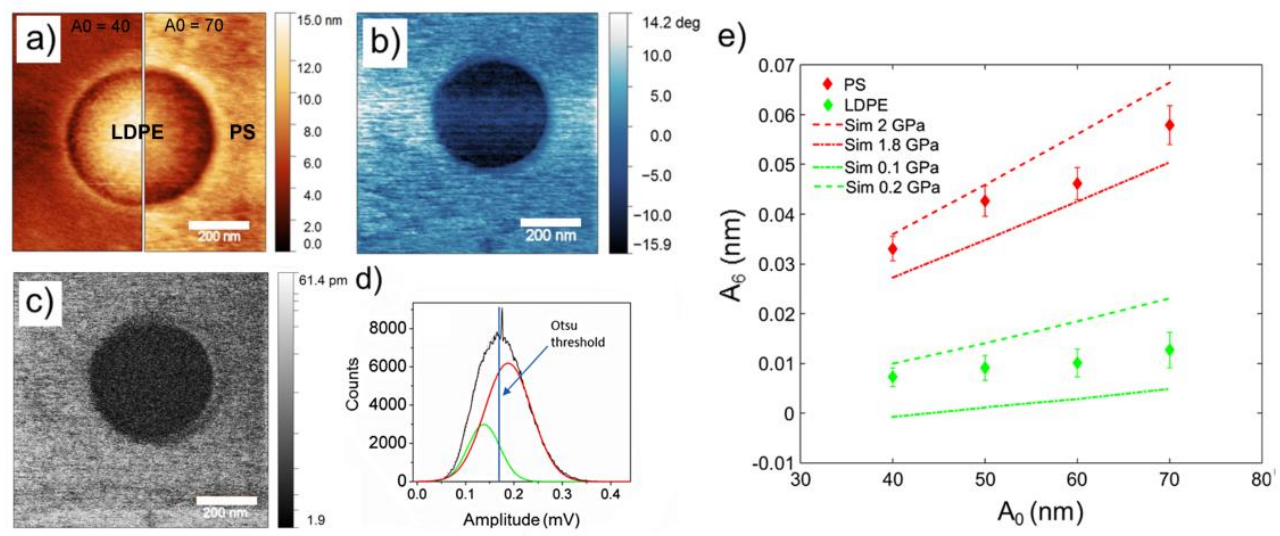

Figure 2. PS-LDPE sample: (a) topography at $A_{0}=40 \mathrm{~nm}$ (left) and $A_{0}=70 \mathrm{~nm}$ (right), (b) phase of the fundamental mode and (c) $A_{6}$ channel images at $A_{0}=70 \mathrm{~nm}$. As $A_{0}$ increases, the force exerted induces an increased deformation in the sample, as visible in the comparison of the two topographies. (d) Example of distribution of the $A_{6}$ channel with a Gaussian fit for both LDPE (continuos green line) and PS (continuos red line) contributions, the vertical blue line representing the Otsu mask threshold value (see text for details). (e) Plot of experimentally determined $A_{6}$ values for different $A_{0}$ values (green diamonds for LDPE and red diamonds for PS) and corresponding simulations for LDPE (discontinuous green line) and PS (continuous red line). The experimental values lie in the given indetermination interval of sample Young's modulus. 


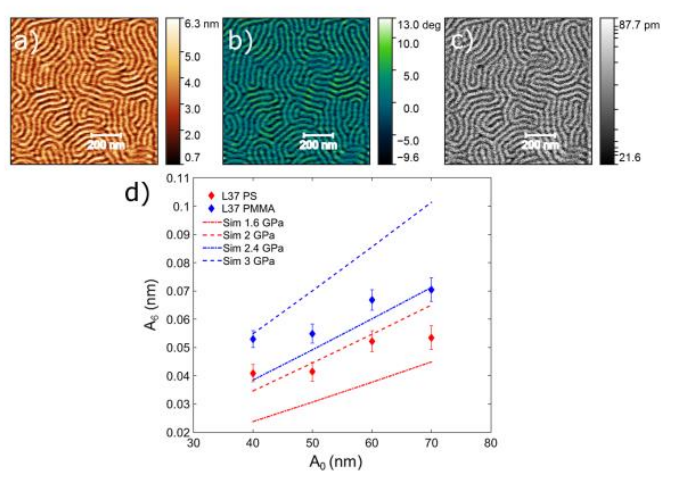

Figure 3. PS-b-PMMA sample: (a) topography, (b) phase and (c) $A_{6}$ channel images taken at $A_{0}=70$ $\mathrm{nm}$. (d) Plot of experimentally determined $A_{6}$ values for different $A_{0}$ values (red diamonds for PS and blue diamonds for PMMA) and corresponding simulations for PS (discontinuous red line) and PMMA (continuous blue line). The experimental values lie in the given indetermination interval of sample Young's modulus.

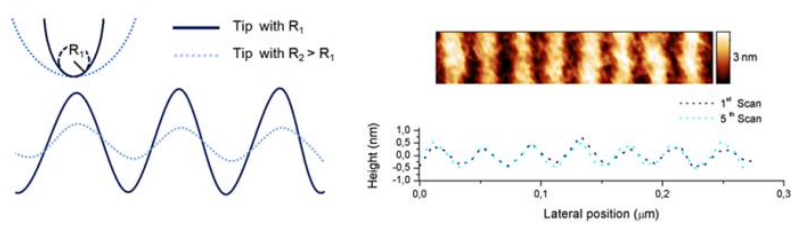

Figure 4 (Left) expected influence of tip blunting on AFM height profile. Scanning at constant load vertical lamellar BCPs produces a certain topographical contrast between the two blocks. As the tip radius increases convolution effects tend to mask such height difference. (Right) in the case of 5 consequent scans at $A_{0}=(40-70) \mathrm{nm}$ and $\mathrm{Asp}=50 \%$ of $\mathrm{A} 0$, no relevant change in the averaged profile of parallel lamellas is observed, thus deducting no significant tip blunting has occurred so far.

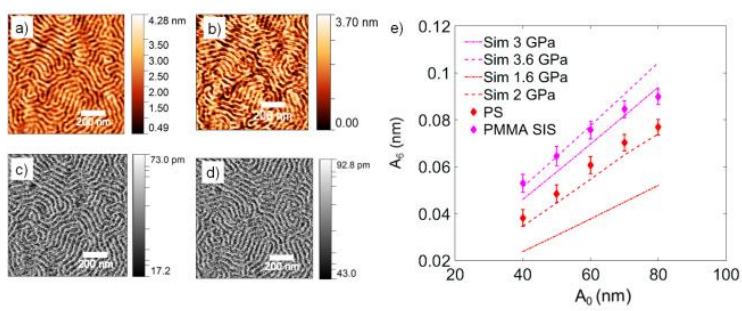

Figure 5. PS-b-PMMA sample with modified PMMA by SIS: topography at (a) $A_{0}=40$ and (b) $A_{0}=$ $70 \mathrm{~nm}$ and $A_{6}$ channel images at (c) $A_{0}=40 \mathrm{~nm}$ and (d) at $A_{0}=70 \mathrm{~nm}$. (e) Plot of experimentally determined $A_{6}$ values for different $A_{0}$ values (red diamonds for PS and magenta diamonds for infiltrated PMMA) and corresponding simulations for PS (discontinuous red line) and infiltrated PMMA (continuous magenta line). Infiltrated phase experimental values match with simulation while non-infiltrated PS phase shows an offset, indicating an apparent higher modulus of PS. 